\title{
SDSS J1553+0056: A BALQSO mimicking a Lyman-break galaxy ${ }^{\star}$
}

\author{
I. Appenzeller ${ }^{1}$, O. Stahl ${ }^{1}$, C. Tapken ${ }^{1}$, D. Mehlert ${ }^{1}$, and S. Noll ${ }^{2}$ \\ 1 Landessternwarte, Königstuhl, 69117 Heidelberg, Germany \\ e-mail: I.Appenzeller@lsw.uni-heidelberg.de \\ 2 Max-Planck-Institut für extraterrestrische Physik, Giessenbachstr., 85741 Garching, Germany
}

Received 13 December 2004/ Accepted 12 January 2005

\begin{abstract}
Using the UVES echelle spectrograph at the ESO VLT we obtained high-resolution $(R=40000)$ spectra of the object SDSS J1553+0056, which has been identified in the literature alternatively as a high-redshift quasar or as a Lyman-break galaxy (LBG). Although low-resolution spectra of SDSS J1553+0056 closely resemble those of LBGs, our high-resolution spectra allow us to identify this object unambiguously as a LoBAL quasar, probably belonging to the rare FeLoBALQSO class. Based on our spectrum we discuss how misidentifications of such objects from low-resolution spectra can be avoided.
\end{abstract}

Key words. galaxies: high-redshift - galaxies: starburst - quasars: absorption lines - quasars: general

\section{Introduction}

In addition to providing a large sample of galaxy spectra, the Sloan Digital Sky Survey (York et al. 2000) has up to now resulted in more than 50000 low-resolution spectra of quasars. These spectra have been published in various SDSS data releases (Stoughton et al. 2002; Abazajian et al. 2003, 2004) and cataloged by Schneider et al. (2002, 2003). As pointed out by Bentz \& Osmer (2004) and Bentz et al. (2004), the lowresolution spectra of some of the objects classified as quasars by the SDSS pipeline closely resemble spectra normally observed for high-redshift starburst galaxies. Therefore, Bentz et al. (2004) suggest that these objects are either luminous starburst galaxies or very peculiar BALQSOs and argue that some of the high-redshift objects in the SDSS quasar catalog are actually high-redshift galaxies or "Lyman-break galaxies" (LBGs).

If this suggestion is correct, the objects listed by Bentz et al. (2004) would be among the brightest high-redshift starburst galaxies known, both apparently and absolutely. In fact these objects are bright enough to be observed with high spectral resolution using efficient Echelle spectrographs at 8-10 m telescopes. With the single exception of the gravitationally lensed galaxy MS 1512-cB58 (Yee et al. 1996; Pettini et al. 2000, 2002; Savaglio et al. 2002) no high-resolution spectra of Lyman-break galaxies have been published so far, and almost all our knowledge about the structure, physical state, and composition of these objects has been inferred from low-resolution spectra. Therefore, we obtained high-resolution echelle spectra of the $z=2.63$ object SDSS J155359.96+005641.4 from the

^ Based on observations obtained with UVES at the ESO Very Large Telescope, Paranal, Chile (proposal No. 073.A-0386(A)). list of Bentz et al. (2004). The aim of our observations was to clarify the physical nature of SDSS J1553+0056 and to gain information on the physics of such luminous objects showing (at low spectral resolution) Lyman-break-galaxy spectra.

\section{Observations, data reduction, and basic spectroscopic results}

The observations were carried out in service observing mode between April and June 2004 with UVES, the Ultraviolet and Visual Echelle Spectrograph at the Nasmyth platform B of ESO's VLT UT2 (Kueyen) on Cerro Paranal, Chile. For all spectra the red channel and the CD No. 3 were used. In total 8 individual spectra covering the spectral range $4160 \AA-6200 \AA$ and 5 spectra covering the overlapping range $4800 \AA-6820 \AA$ were obtained. Thus, in the rest frame of SDSS J1553+0056 our combined spectra cover the wavelength range 1145-1880 . The projected slit width was 1 arcsec, resulting in a spectral resolution of $\lambda / \Delta \lambda \approx 40000$ which corresponds to a velocity resolution of $7.5 \mathrm{~km} \mathrm{~s}^{-1}$. The exposure times of the individual frames varied between 28 and $50 \mathrm{~min}$, the total integration time for the spectral overlap region amounted to about $10.5 \mathrm{~h}$. Three of the individual exposures with a total exposure time of more than $2 \mathrm{~h}$ were, however, obtained under poor observing conditions (outside specifications) and, therefore, contain little information. The average FWHM seeing for the observations was about 0.8 arcsec, and extrema were 0.5 and 1.8 arcsec.

Because of the low signal in parts of our spectra the standard ESO pipeline software for UVES turned out to be unsuitable for our data; therefore, the reduction was carried out using special software initially developed by us for the reduction 

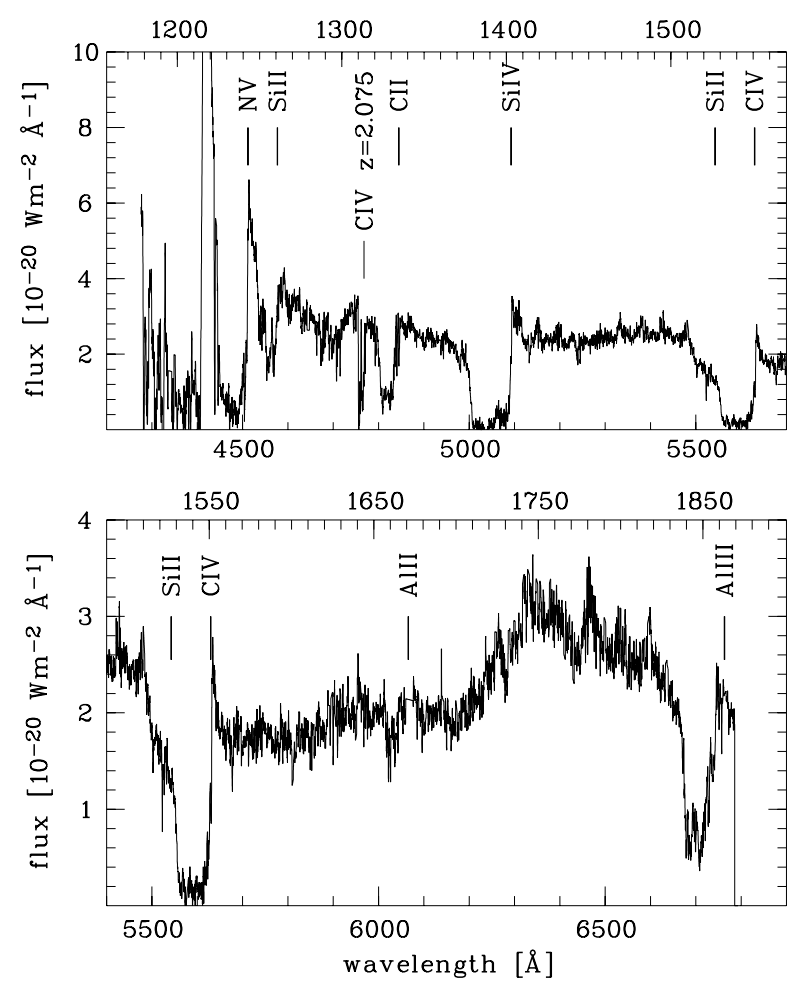

Fig. 1. The complete spectrum of SDSS J1553+0056. The top axis plots rest-frame wavelengths assuming a systemic redshift of $z=$ 2.6327. The bottom axis gives the observed wavelengths.

of FEROS echelle spectra (Stahl et al. 1999). In a first step we carried out a two-dimensional reduction of the spectral frames. Then the echelle orders were merged and one-dimensional spectra extracted. For this procedure all spectral regions with defects or too low an $\mathrm{S} / \mathrm{N}$ were omitted. The wavelength scale was then converted to heliocentric vacuum wavelengths, and spectra from the individual exposures were co-added with equal weight. Outside the absorption troughs the resulting merged spectrum has a continuum signal-to-noise ratio (per spectral resolution element) of about 10 .

Since the spectra were taken under variable observing conditions, no direct flux calibration of our UVES spectra was attempted. Instead we used the archive SDSS spectrum of SDSS J1553+0056 to correct for the UVES instrumental response and to convert the spectrum to flux units. The corresponding total flux-calibrated spectrum is reproduced in Fig. 1. For plotting this figure the effective spectral resolution has been reduced to $R \approx 5000$ using a median filter.

\section{The physical nature of SDSS J1553+0056}

As shown by Fig. 1 the spectrum of SDSS J1553+0056 is dominated by broad absorption troughs of resonance lines and blends. The shapes (see Fig. 1) and widths (see Table 1) of these lines are clearly reminiscent of the spectra of the Broad Absorption Line (BAL) quasars (see e.g. Weymann et al. 1991; Hall et al. 2002; Brunner et al. 2003; Reichard et al. 2003). But apart from Ly $\alpha$ the spectrum of SDSS J1553+0056 shows no strong emission lines. Absorptions troughs corresponding to low-ionization resonance lines are prominent. Thus,
Table 1. Full widths (at continuum level) of the absorption troughs of resonance lines. For the doublets the measured width was reduced by the doublet separation to get values corresponding approximately to the individual doublet components.

\begin{tabular}{llll}
\hline \hline Ion & $\lambda_{\text {lab,vac }}(\AA)$ & Trough width $\left(\mathrm{km} \mathrm{s}^{-1}\right)$ & Remark \\
\hline N V & $1238.82,1242.80$ & $4200 ?$ & bl. Ly $\alpha$ \\
Si II & 1260.42 & 2994 & \\
C II & 1334.53 & 2413 & \\
Si IV & $1393.76,1402.77$ & 3997 & \\
C IV & $1548.20,1550.77$ & 4624 & bl. Si II (UV2) \\
Al II & 1670.79 & 2471 & bl. Fe II (UV40 - 42) \\
Al III & $1854.72,1862.79$ & 2158 & \\
\hline
\end{tabular}

SDSS J1553+0056 obviously belongs to the LoBAL sub-class of the BALQSOs as defined by Voit et al. (1993). The red edges (i.e. wavelengths corresponding to the onset of the sharp drop of the flux in the red wings of the troughs) of the Ly $\alpha$, NV $\lambda$ 1242.80, Si IV 1402.77, and C IV 1550.77 absorption troughs correspond to a common redshift of $2.6327 \pm 0.0002$. In the following we shall assume, therefore, that this value approximates the systemic redshift of this BALQSO. All velocities listed below are given in relation to this redshift. As shown in Fig. 2, the red edges of the low-ionization troughs have smaller redshifts. But all low-ionization lines show narrow $\left(F W H M \leq 40 \mathrm{~km} \mathrm{~s}^{-1}\right)$ line components with redshifts between the redshift of the high-ionization and the low-ionization red edges.

In the case of resonance doublets, the edge profiles plotted in Fig. 2 correspond to the red doublet components. For $\mathrm{Si}$ IV there is no significant overlap by the corresponding blue doublet component in the velocity range given Fig. 2. In the case of Al III, N V, and C IV the doublet components overlap for $v \leq-1300, \leq-960$, and $\leq-500 \mathrm{~km} \mathrm{~s}^{-1}$, respectively. Because of these overlaps and the low flux inside the troughs, the present discussion is restricted to the red edges.

Some of the narrow low-ionization lines mentioned above are visible in Fig. 2. Since part of these narrow lines in Fig. 2 are observed with the same profiles in different echelle orders these features definitely are not artifacts. In the case of the Al III resonance doublet, the large doublet separation and the relatively high residual flux in the absorption trough (see Fig. 1) allowed us to compare the edge profiles and the pattern of sharp absorption lines of the two doublet components. As expected, the edge profiles and the sharp line profiles of the two doublet components turned out to be identical. On the other hand Fig. 2 and Table 1 show that for lines not belonging to the same ion the edge profiles the sharp components of the low-ionization lines and the widths of the trough profiles are clearly different. There are some common properties, however, most profiles show absorption features at -300 to $-360 \mathrm{~km} \mathrm{~s}^{-1}$ and -590 to $-650 \mathrm{~km} \mathrm{~s}^{-1}$. These features are narrow in the low-ionization lines and broader for the high-ionization species. The mean velocities of these velocity components, as derived from averaging the velocities measured for the individual lines, are indicated in Fig. 2 by dotted vertical lines. All troughs with sufficient rest flux show a "window" of less deep trough absorption near $-760 \mathrm{~km} \mathrm{~s}^{-1}$. 


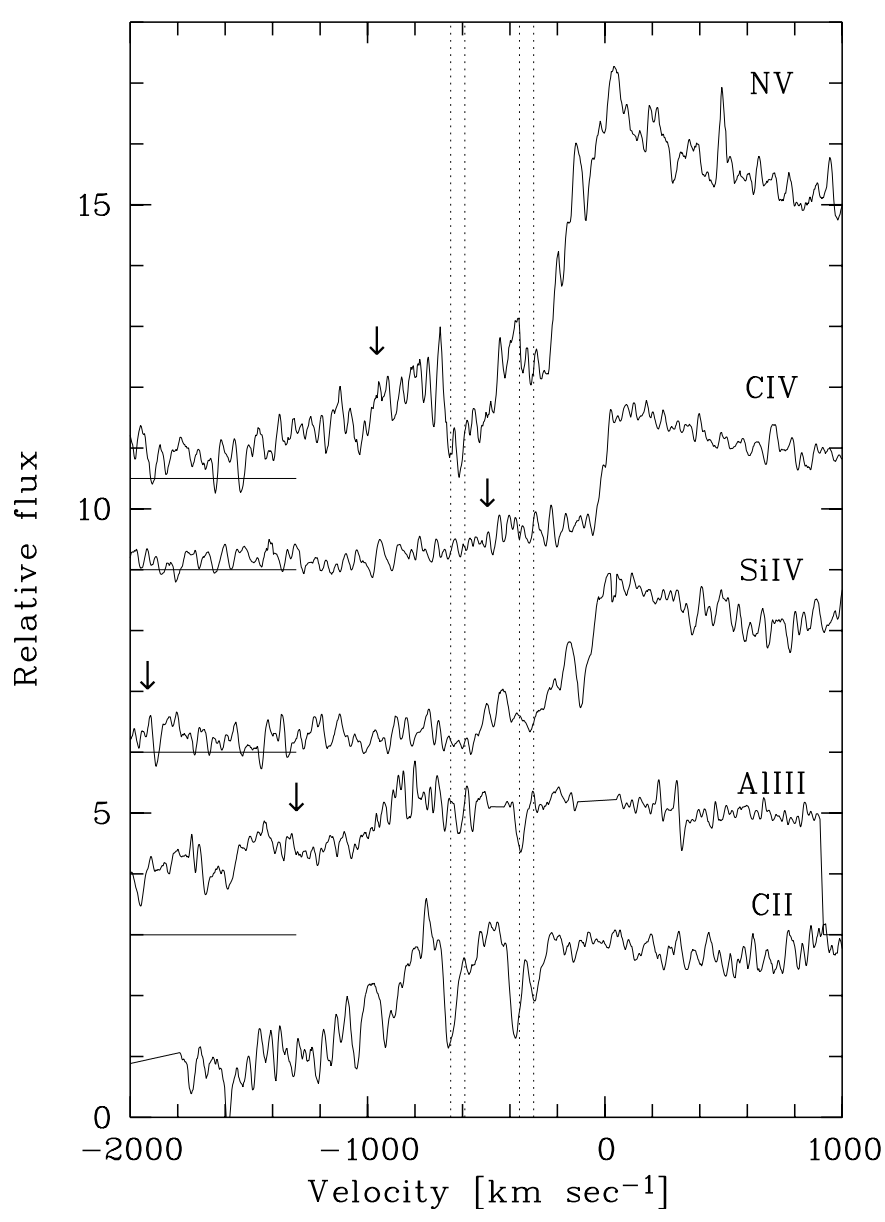

Fig. 2. Profiles of the red edges of several resonance-line absorption troughs. The velocity scale assumes a systemic redshift of 2.6327 . In the case of doublets the velocity refers to the red doublet component. The $(v=0)$ positions of the blue doublet components are indicated by vertical arrows. In the case of C II the velocity scale corresponds to the mean wavelength of the two fine structure components $\mathrm{C}_{\mathrm{II}^{*}} \lambda \lambda$ 1335.66, 1335.71 of $\mathrm{C}$ II (UV1). Features caused by the ground state $\lambda 1334.53$ line of this multiplet are shifted (by about $260 \mathrm{~km} \mathrm{~s}^{-1}$ ) to apparently more negative velocities. As a result the $-360 \mathrm{~km} \mathrm{~s}^{-1}$ component of the $1334.53 \AA$ line overlaps with the $-650 \mathrm{~km} \mathrm{~s}^{-1}$ component of $\mathrm{C} \mathrm{II}^{*}$, while the $-650 \mathrm{~km} \mathrm{~s}^{-1}$ feature of the 1334.53 line occurs at the apparent velocity $-910 \mathrm{~km} \mathrm{~s}^{-1}$. The apparent common absorption features mentioned in the text are indicated by dotted vertical lines.

It cannot be excluded that the observed narrow absorption lines described above are caused by foreground metal absorption systems with slightly smaller redshifts. However, the absence of corresponding narrow features at the high-ionization lines, which only show broader features, seems to argue against this assumption. Therefore, we suggest that the sharp lines form in (still) relatively cool and dense interstellar clouds of the ISM of the QSO host galaxy which so far have been shielded from the central source and have not yet been significantly accelerated by radiation from the central source. If this assumption is correct, the observed structure of the red edges of resonance lines and the presence of narrow line components with common velocities slightly blueward of the high-ionization red edge velocity both seem to support suggestions that BALQSO

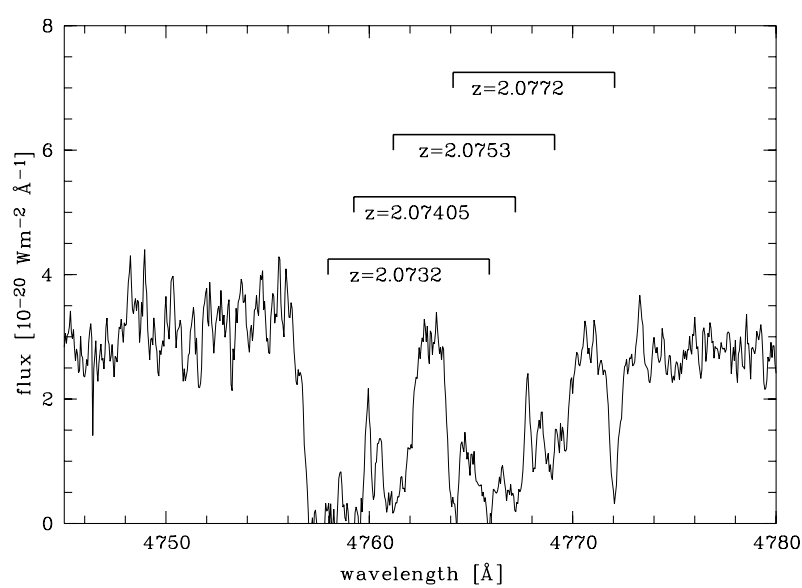

Fig. 3. Profile of the complex $2.0729<z<2.0772$ foreground C IV doublet absorption system.

troughs result from the superposition of individual absorption lines originating in a modest number of absorbing gas clouds that are accelerated (and eventually dispersed) by a recently switched on AGN (see e.g. Williams et al. 1999; Hall et al. 2002). The observed profile differences correlated with the ionization stage indicate that the absorbing clouds at this evolutionary stage differ greatly in their physical parameters and in their internal radiation and velocity fields.

In addition to the narrow resonance absorption lines with redshifts close to the assumed systemic velocity, at least three C IV absorption systems with smaller redshifts (at $z=1.8679$, 2.0413, and 2.0729-2.0772) are present. The complex profile of the C IV doublet of the 2.0729-2.0772 system is reproduced in Fig. 3. The $\mathrm{Al} \mathrm{II} \mathrm{and} \mathrm{Al}$ III resonance absorption lines of this system are also present in our spectra.

Apart from the fine structure lines of C II (UV1) mentioned in Fig. 2, no other narrow absorption lines originating from excited atomic energy levels could be detected. Moreover, a thorough search for lines typical of the photospheric spectra of hot stars (such as O IV 1343.35, C III 1427.85, and S V $1501.76 \AA$ at $z \approx 2.633$ ) was not successful, so that integrated stellar light from the QSO's host galaxy cannot contribute much to the observed flux. The depth of the C IV and Si IV trough shows that coverage of the continuum source by the absorbing clouds must be close to complete.

As shown by Fig. 1 the red part of our spectrum contains a region of high flux with some apparent peaks in the wavelength range 6300-6600 $\AA$ (corresponding to $\approx 1740$ $1800 \AA$ in the QSO's restframe) and a region of low flux between 5600 and $6300 \AA$ (1550-1740 $\AA$, restframe). These SED features are qualitatively similar to those observed in certain FeLoBALQSOs, such as SDSS 0318-0600 (Hall et al. 2002), PSS J0052+2405 (Brunner et al. 2003), in the composite FeLoBAL spectrum presented by Reichard et al. (2003, Fig. 1), and in the BALQSO FDF-6233 (Noll et al. 2004). As pointed out by Hall et al. (2002) these features can be explained by the presence of of Ni II and Fe II lines originating in part from excited atomic energy levels. A detailed comparison with the UV multiplet tables shows that modulation of the pseudo-continuum of SDSS J1553+0056 in the region 
5600-6300 ̊ is qualitatively consistent with a superposition of merging broad absorption lines originating from Si II (UV1), Ni II (UV2 to UV5), and Fe II (UV multiplets 8, 9, and 3846). At least part of the apparent steps in the flux distribution seem to coincide with the red limits of some of these multiplets. But no narrow absorption components originating from excited Fe II energy levels could be detected so the individual lines appear to produce weak absorption troughs of unknown width.

The possible presence of broad Fe II absorption from excited energy levels suggest that SDSS J1553+0056 is a member of the rare FeLoBALQSO class (Hazard et al. 1987; Becker et al. 1997, 2000; Menou et al. 2001). But our UVES spectra do not contain those lines of the Fe II multiplets UV1 and UV2 that define this class. The low-resolution spectrum of SDSS J1553+0056 in the SDSS archives includes the Fe II (UV2) multiplet. On this spectrum an Fe II (UV2) absorption trough appears to be present, but the corresponding spectral region coincides with telluric $\mathrm{OH}$ emission. Hence, a definite confirmation of the FeLoBALQSO character of SDSS J1553+0056 will require higher resolution spectra in the red $(\lambda>7000 \AA)$ spectral range.

Compared to other FeLoBALQSOs, our target shows little evidence for dust reddening. The overall SED in the observed wavelength range is bluer than the dereddened SED of the FeLoBALQSO SDSS 0318-0600 (Hall et al. 2002).

\section{Comparison with galaxy spectra and implications for LBG searches}

Bentz et al. (2004) suggested that SDSS J1553+0056 is a Lyman Break Galaxy on the basis of the high-ionization resonance absorption lines observed (thought to be stellar wind features) and the apparent presence of narrow (ISM) absorption lines of Si II, O I, and C II, which are prominent in LBG spectra. Moreover, for a QSO the SED of SDSS J1553+0056 is unusually flat and resembles that of a slightly reddened LBG, and the Ly $\alpha$ line of SDSS J1553+0056 appears rather narrow for a QSO.

Our high resolution spectra show that the narrow appearance of the Ly $\alpha$ line is due to absorption by the Ly $\alpha$ trough in the blue wing and by the $\mathrm{N} v$ trough in the red wing. The other emission lines appear intrinsically weak in SDSS J1553+0056 and are partly absorbed by their own troughs. These troughs show no or little detachment from the emission features. Moreover, low-ionization line emission is weakened on the low-resolution spectrum by unresolved narrow absorption features. That the BAL absorption troughs have been misidentified as stellar wind features is obviously due to their modest width, which is near the lower limit found for BALQSOs. However, the observed trough widths are still inside the range of 2000-20000 $\mathrm{km} \mathrm{s}^{-1}$ typical for this class of objects. The particularly low widths of the $\mathrm{C}$ II and $\mathrm{Si}$ II line troughs resulted in their misidentification as ISM features. And the foreground $z \approx 2.07 \mathrm{C}$ IV system appearing by chance coincidence at the corresponding wavelength mimicked the presence of a sharp ISM O I absorption feature.
LoBALQSOs, in particular FeLoBALQSOs, are relatively rare objects (see e.g. Menou et al. 2001). However the surface density on the sky of high-redshift LBGs is also small, so at least at the high absolute luminosity end, LBG identifications from low resolution spectra can be affected by objects similar to SDSS J1553+0056. As pointed out by Bentz et al. (2004) identifications of high-luminosity LBGs determine the galaxy luminosity functions at high redshift, with significant cosmological implications. Thus, it is important to avoid such misidentification.

On the basis of our results the most reliable separation between objects such as SDSS J1553+0056 and LBGs from low resolution spectra appears to be the continuum features caused by excited Fe II levels in the restframe 1550 $1800 \AA$ range, as such features are never observed in LBGs. Unfortunately evaluation of these continuum features, while possible at very low spectral resolution, requires very accurate flux calibration of the spectra, which is difficult for faint objects. Moreover, in QSOs this feature seems to be restricted to the rare FeLoBALQSOs.

If the high-ionization absorption troughs of a BALQSO are as narrow as or narrower than in the case of SDSS J1553+0056 at low spectral resolution misidentification with stellar wind lines which in certain types of hot stars can reach similar widths probably cannot be excluded. However, comparison of our UVES spectrum with LBG spectra indicates that the strength of the C II (UV1) absorption in the low-resolution spectrum of SDSS J1553+0056 ( $\left.E W_{\text {rest }}>6 \AA\right)$ could have been used to determine the nature of this object. In LBGs this absorption is normally caused by ISM absorption. Such ISM lines are usually narrow, which results in a low equivalent width ( $\leq 4 \AA$ in LBGs), independent of the resolution. Thus, the high equivalent widths of low-ionization resonance lines, such as the strong C II (UV1) absorption observed in the low-resolution spectrum of SDSS J1553+0056 are telltale signs of the presence of the absorption troughs characteristic of LoBALQSOs.

Finally, we note that in composite spectra of representative samples of LBGs (see e.g. Shapley et al. 2003; and Noll et al. 2004), the N V stellar wind absorption feature is never as deep as observed in SDSS J1553+0056. Therefore, all LBG spectra showing such strong blueshifted $\mathrm{N} \mathrm{V}$ absorption should be checked for the presence of other BALQSO indicators.

Application of the above criteria indicates that in addition to SDSS J1553+0056 at least three more of the 6 objects in the Bentz et al. (2004) list could possibly be BALQSOs. Thus, it appears desirable to clarify the nature of these objects by means of higher resolution spectroscopy before conclusions are drawn from their tentative identifications as LBGs.

\section{Conclusions}

Our high-resolution spectroscopic observations of SDSS J1553+0056 have shown that this object is not an LBG but instead a LoBALQSO that probably belongs to the FeLoBALQSO class. Compared to other BALQSOs it has weak emission lines, relatively narrow absorption troughs; and it shows (relative to other FeLoBALQSOs) little evidence of dust reddening. Because of these properties the 
low-resolution spectrum of SDSS J1553+0056 resembles those of high-redshift starburst galaxies. However, there is no evidence for any stellar light contribution to the observed flux from SDSS J1553+0056. From a comparison of our spectra with mean LBG spectra we conclude that on low-resolution spectra objects similar to SDSS J1553+0056 show relative to LBGs (1) a significantly different flux distribution in the (rest-frame) wavelength range 1550-1800 $\AA$; (2) stronger low-ionization resonance absorption features; and (3) stronger $\mathrm{N} \mathrm{V}$ resonance absorption. If any of these properties is observed in a low-resolution LBG spectrum, the object could be a misidentified BALQSO. However, because of the large range of properties of BALQSOs, the absence of any of these signs does not guarantee that the observed object is a bona fide LBG. Particularly at the high luminosity end of the LBG luminosity function, there is obviously the danger of BALQSOs polluting low-resolution spectroscopy based LBG samples.

Acknowledgements. It is a pleasure to thank the ESO Paranal Observatory staff for carrying out for us the service mode observations on which this paper is based, and Dr. Patrick Osmer for reading our manuscript and for valuable comments. This work was supported by the German Science Foundation (DFG, SFB 439).

\section{References}

Abazajian, K., Adelman-McCarthy, J. K., Agüeros, M. A., et al. 2003, AJ, 126, 2081

Abazajian, K., Adelman-McCarthy, J. K., Agüeros, M. A., et al. 2004, AJ, 128, 502

Becker, R. H., Gregg, M. D., Hook, I. M., et al. 1997, ApJ, 479, L93
Becker, R. H., White, R. L., Gregg, M. D., et al. 2000, ApJ, 538, 72 Bentz, M. C., \& Osmer, P. S. 2004, AJ, 127, 576

Bentz, M. C., Osmer, P. S., \& Weinberg, D. H. 2004, ApJ, 600, L19

Brunner, R. J., Hall, P. B., Djorgovski, S. G., et al. 2003, AJ, 126, 53

Hall, P. B., Anderson, S. F., Strauss, M. A., et al. 2002, ApJS, 141, 267

Hazard, C., McMahon, R. G., Webb, J. K., \& Morton, D. C. 1987, ApJ, 323, 263

Menou, K., Vanden Berk, D. E., Ivezić, Ž., et al. 2001, ApJ, 561, 645

Noll, S., Mehlert, D., Appenzeller, I., et al. 2004, A\&A, 418, 885

Pettini, M., Rix, S. A., Steidel, C. C., et al. 2002, ApJ, 569, 742

Pettini, M., Steidel, C. C., Adelberger, K. L., Dickinson, M., \& Giavalisco, M. 2000, ApJ, 528, 96

Reichard, T. A., Richards, G. T., Schneider, D. P., et al. 2003, AJ, 125, 1711

Savaglio, S., Panagia, N., \& Padovani, P. 2002, ApJ, 567, 702

Schneider, D. P., Fan, X., Hall, P. B., et al. 2003, AJ, 126, 2579

Schneider, D. P., Richards, G. T., Fan, X., et al. 2002, AJ, 123, 567

Shapley, A. E., Steidel, C. C., Pettini, M., \& Adelberger, K. L. 2003, ApJ, 588, 65

Stahl, O., Kaufer, A., \& Tubbesing, S. 1999, in Optical and Infrared Spectroscopy of Circumstellar Matter, ed. E. W. Guenther, B. Stecklum, \& S. Klose, ASP Conf. Ser., 188, 331

Stoughton, C., Lupton, R. H., Bernardi, M., et al. 2002, AJ, 123, 485

Voit, G. M., Weymann, R. J., \& Korista, K. T. 1993, ApJ, 413, 95

Weymann, R. J., Morris, S. L., Foltz, C. B., \& Hewett, P. C. 1991, ApJ, 373,23

Williams, R. J. R., Baker, A. C., \& Perry, J. J. 1999, MNRAS, 310, 913

Yee, H. K. C., Ellingson, E., Bechtold, J., Carlberg, R. G., \& Cuillandre, J.-C. 1996, AJ, 111, 1783

York, D. G., Adelman, J., Anderson, J. E., et al. 2000, AJ, 120, 1579 